\title{
Physical models of asteroids from sparse photometric data
}

\author{
Josef Ďurech $^{1,3}$, Petr Scheirich ${ }^{2}$, Mikko Kaasalainen ${ }^{3}$, Tommy Grav ${ }^{4}$, \\ Robert Jedicke ${ }^{4}$ and Larry Denneau ${ }^{4}$ \\ ${ }^{1}$ Astronomical Institute, Charles University in Prague, \\ V Holešovičkách 2, 18000, Prague, Czech Republic, \\ email: durech@sirrah.troja.mff.cuni.cz \\ ${ }^{2}$ Astronomical Institute, Academy of Sciences of the Czech Republic, \\ Fričova 1, 25165 Ondřejov, Czech Republic \\ ${ }^{3}$ Department of Mathematics and Statistics, Rolf Nevanlinna Institute, University of Helsinki, \\ P.O. Box 68, 00014 Helsinki, Finland \\ ${ }^{4}$ Institute for Astronomy, University of Hawaii, \\ 2680 Woodlawn Drive, Honolulu, Hawaii, 96822, USA
}

\begin{abstract}
We present an overview of our work on shape and spin state determination of asteroids from photometric data sparse in time. Our results are based on simulations that were performed using realistic shape and light-scattering models and time sequences that will be provided by Pan-STARRS (Panoramic Survey Telescope and Rapid Response System). We show some typical examples of physical model reconstruction of main belt and near-Earth asteroids and discuss the lightcurve inversion of slow and fast rotators, binary asteroids and tumbling asteroids. We emphasize the scientific potential of sparse photometric data to produce models of a large number of asteroids within the next few years.
\end{abstract}

Keywords. survey; photometry; asteroid, lightcurves; asteroid, shape

\section{Introduction}

Astronomers have observed asteroid lightcurves - brightness variations as a function of time - for many decades. Since a typical asteroid rotates with a period of hours, a substantial part of its whole rotation cycle can be covered during one night. A set of lightcurves from several apparitions with different observing geometries contains so much information about the spin state and the shape of an asteroid, that those physical parameters can be unambiguously derived using lightcurve inversion. The lightcurve inversion method developed by Kaasalainen \& Torppa (2001) and Kaasalainen et al. (2001) has led to about a hundred asteroid models (Kaasalainen et al. 2002b, 2004; Torppa et al. 2003) and its reliability has been proven by ground truths from, e.g., Kaasalainen et al. (2001, 2005) and Marchis et al. (2006).

However, it is not necessary to have lightcurves that densely cover the rotational phase for deriving a plausible physical model of an asteroid. As has been shown by Kaasalainen (2004), calibrated brightness measurements from several years that are sparse in time are fully sufficient. This approach is much more time efficient than the standard approach of observing dense lightcurves of selected targets. Future asteroid surveys will provide us with a huge amount of accurate sparse photometric data and we will have the opportunity to reconstruct physical models of a substantial part of the asteroid population. Lightcurve inversion of sparse photometric data will be an essential method for asteroid shape and spin state modelling in the near future. 

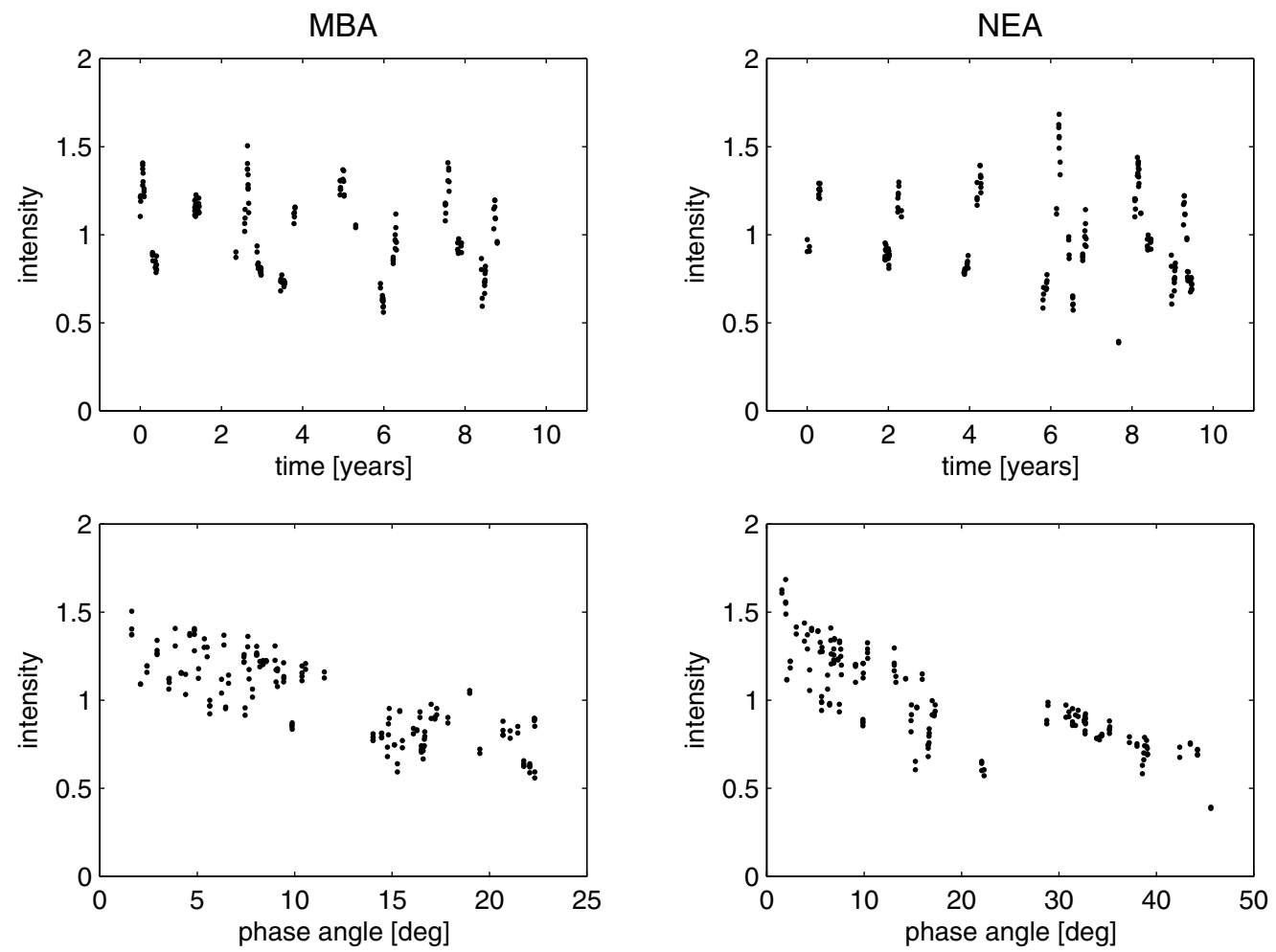

Figure 1. Typical Pan-STARRS observations of a MBA (left) and a NEA (right) shown as a time sequence covering ten years (top) and as a brightness vs. solar phase angle plot (bottom). The brightness is reduced to unit distances from the Earth and the Sun.

In the following text, we often refer to results presented by Kaasalainen \& Durech, Inverse problems of NEO photometry: Imaging the NEO population, in this proceedings book (hereafter KD). This paper gives a general overview about methods for modelling asteroid physical parameters and about the lightcurve inversion in particular.

So far, sparse data have been available only as a by-product of astrometric observations. Such data usually suffer from large systematic errors and noise and cannot be used alone (see KD, Sect. 4.1). Future photometric surveys will reach much better photometric accuracy and the lightcurve inversion applied to such accurate data will lead to unambiguous and reliable physical models.

We summarize the results of sparse data analysis in this paper that were obtained using simulations based on Pan-STARRS (Panoramic Survey Telescope and Rapid Response System) cadences. Pan-STARRS is an ongoing project at the Institute for Astronomy of the University of Hawaii, the first light is scheduled for early 2007. It is the first project that will provide sparse photometry with sufficiently low calibration errors.

Another source of accurate photometric data sparse in time will be the Gaia space mission. Although its lifetime will be shorter than for ground-based surveys, it will observe at small solar elongations, thus covering a wider range of ecliptic longitudes in shorter time. 


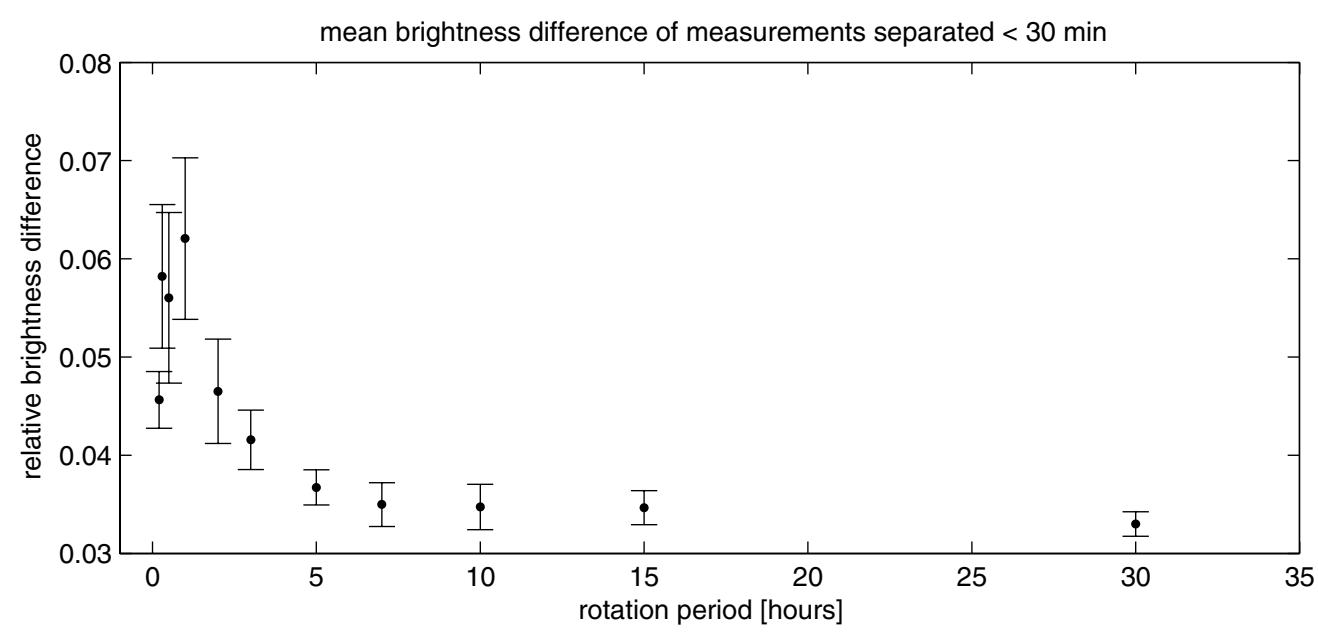

Figure 2. The mean relative brightness difference between observations separated by less than $30 \mathrm{~min}$ as a function of the rotation period.

\section{Photometry sparse in time}

Contrary to a set of individual lightcurves that densely cover a substantial part of the full rotation cycle, sparse data sets consist of individual brightness measurements, typically only one or a few points per night. Sparse photometry has to be calibrated in the absolute scale - we need to directly compare brightness measurements with each other on the time scale of many years. Examples of synthetic sparse data sets are shown in Fig. 1. The plots were generated using the Pan-STARRS observing scheduler, a realistic shape model and Hapke's light-scattering model. The brightness was reduced to unit distances from the Earth and the Sun. The main belt asteroid (MBA) data consist of 171 points and the near-Earth asteroid (NEA) data consist of 134 points. Both sets cover ten years of observation. The scatter in the phase angle plots is caused by asteroid's rotation and (to some extent) by a Gaussian noise of $3 \%$ that was added to the synthetic data. There are two groups of points at the phase angle plot of the MBA separated by a gap at $\alpha \sim 13^{\circ}$ corresponding to observations near the opposition and to sweet spots $\dagger$ observations at $\alpha \gtrsim 15^{\circ}$. Naturally, NEA observations reach much larger solar phase angles.

An important feature of the Pan-STARRS observing strategy is that each field of sky will be observed twice each night after about 15 min. This fact enables us to make some preliminary estimations about asteroid's rotation rate before actual modelling. If an asteroid rotates slowly, than its lightcurve variations at the time scale of $\sim 15 \mathrm{~min}$ are very small (except when the object is extremely elongated or there are very sharp features in its lightcurve). On the other hand, when the rotation period is comparable with the time baseline of the subsequent measurements, the typical dispersion of such measurements is as large as the lightcurve amplitude. An example of this behaviour is shown in Fig. 2 . We generated many lightcurves for various combinations of cadence/shape/pole/period and then computed the mean difference between all subsequent measurements separated by less than $30 \mathrm{~min}$. The plot clearly shows that the brightness difference between close pairs is only slightly larger than the noise of the data (which was $3 \%$ ) for rotation periods above $\sim 5 \mathrm{hr}$. It means that if the mean brightness difference between close observations

$\dagger$ Sweet spots are sky regions near quadrature with $|\beta|<10^{\circ}$ and $60^{\circ}<|l|<90^{\circ}$, where $\beta$ is the ecliptic latitude and $l$ is the solar elongation. 

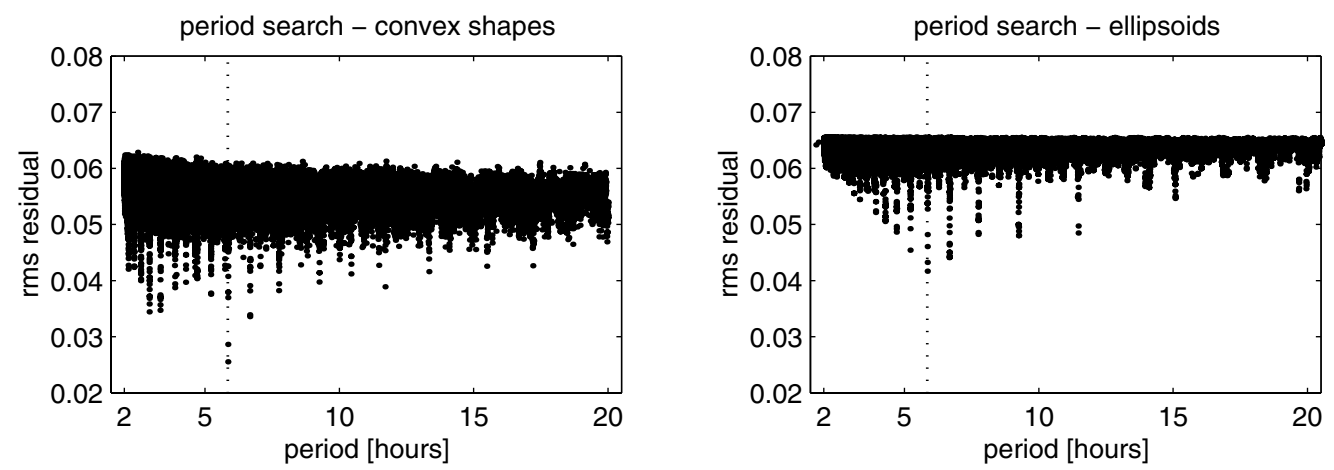

Figure 3. Period search results using convex shapes (left) and triaxial geometrically scattering ellipsoids (right). The correct period is marked with the dotted vertical line and it gives the best fit in both cases.

is significantly larger than the expected noise, the rotation period is not likely to be longer than 10-20 times the mean separation between those observations.

\section{Lightcurve inversion of sparse data}

The process of lightcurve inversion of sparse data sets is basically the same as the lightcurve inversion of ordinary lightcurves (Kaasalainen et al. 2001). The specific approach to the sparse data inversion was described by Kaasalainen (2004). As has been shown by Kaasalainen (2004) and Durech et al. (2006), the crucial parameters affecting results of sparse photometry inversion (uniqueness and stability of the solution) are calibration errors, the number of points in the data set, the time span of observations, and the shape of the asteroid. Observations should be carried out at the widest possible range of solar phase angle and solar elongation in order to provide good sampling of viewing/illumination geometry. We can say in general that if the noise is not higher than $\sim 5 \%$, if there are more than $\sim 100$ points spread over at least five years, and if the shape is not too spheroidal, then a unique physical model can be derived.

We used Gaussian random shapes (Muinonen 1996) and Hapke's scattering model with uniform albedo for synthetic data generation. After adding a certain level of noise, we inverted the data using the empirical light-scattering model of Kaasalainen et al. (2001). As is discussed in KD, Sect. 2.2, the convex inversion guarantees that the solution is not very sensitive to the light-scattering model we use. Another advantage of the convex inversion method is the fact, that any significant asymmetric albedo variegation over the surface reveals itself as the violation of the convexity condition (see KD, Sect. 2.1, for more detailed discussion).

\subsection{Period}

The most important part of the sparse photometry inversion is the correct determination of the rotation period. Unlike in the case of ordinary lightcurves, the period is not 'visible' in the data and it must be searched over a very wide interval of expected values in every single case. Any formal time series analysis will fail to find the period because a typical sampling interval of the data is much longer than the period. However, the correct solution can be found if a realistic physical model is used.

The rotation period is searched by scanning a wide range of periods (typically $2-24 \mathrm{hr}$ ) with the step slightly shorter than $0.5 P^{2} / \Delta T$, where $P$ is the trial rotation period and $\Delta T$ is the length of the interval covered by observations. This step in period corresponds 

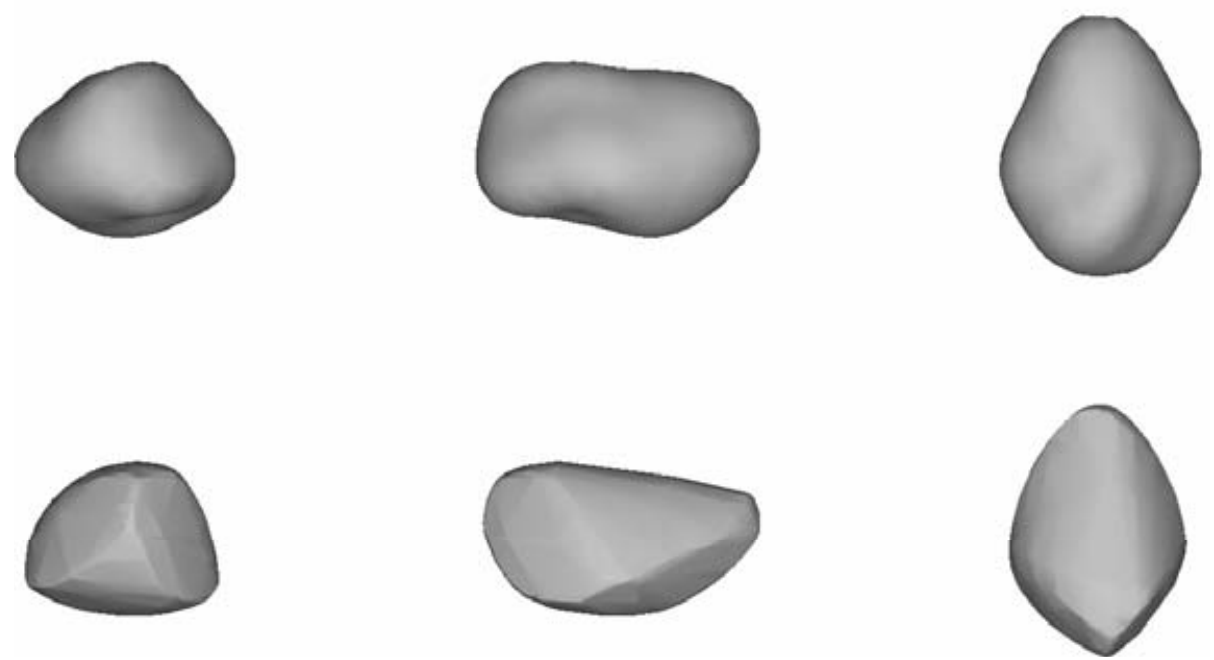

Figure 4. Original shape (top) and the model (bottom) of a MBA asteroid. There are two equatorial views $90^{\circ}$ apart (left and middle) and one pole-one view (right) for both shapes. Pole direction of the original shape and of the model is $\left(348^{\circ},-19^{\circ}\right)$ and $\left(347^{\circ},-15^{\circ}\right)$, respectively. The model rotates with the same period as the original shape, $P=17.6669$ hours.

to a typical separation of local minima in the period parameter space (Kaasalainen 2004). When the best period is found, a refined pole/shape solution is computed using a dense initial pole grid. The period search is a time consuming process because the number of trial periods is very large, typically $\sim 100000$ for $\Delta T=10$ years.

The information about the period hidden in the data is so strong, that it is possible to use a very simple and inaccurate physical model of a geometrically scattering triaxial ellipsoid (rotating along its shortest axis) and still the correct solution stands out clearly. Moreover, the integrated brightness of such model can be computed analytically (see KD, eqs. (3.6)-(3.9)), which makes the period search process about hundred times faster. There is an example of a period search in Fig. 3. The rms residual of the fit is plotted against the period. Although the ellipsoidal model cannot fit the data down to the noise level $(3 \%)$, the correct period (or more possible candidates) can be found and then the physical model can be derived using convex lightcurve inversion.

Our simulations show that the shape of an asteroid is crucial for the period determination. Shapes that are only slightly elongated have only small lightcurve amplitudes that are comparable to the noise and any unique period cannot be found. On the other hand, elongated asteroids have large lightcurve amplitudes and the correct period can be detected even from noisy data.

\subsection{Pole and shape}

The pole direction and the corresponding shape model can be derived once a unique period solution is found. The error in the pole direction is usually not larger than $10^{\circ}$ of arc. There are two solutions of the pole direction for a given period in many cases. They have similar values of the ecliptic latitude $\beta$ and the ecliptic longitudes $\lambda$ are $180^{\circ}$ apart. This ambiguity is inevitable for disk-integrated photometry of targets that orbit near the plane of ecliptic. See KD, Sect. 2.1, or Kaasalainen \& Lamberg (2006) for more detailed explanation. An example of shape reconstruction is shown in Fig. 4. We used simulated Pan-STARRS cadence of a MBA, a nonconvex shape and Hapke's scattering 

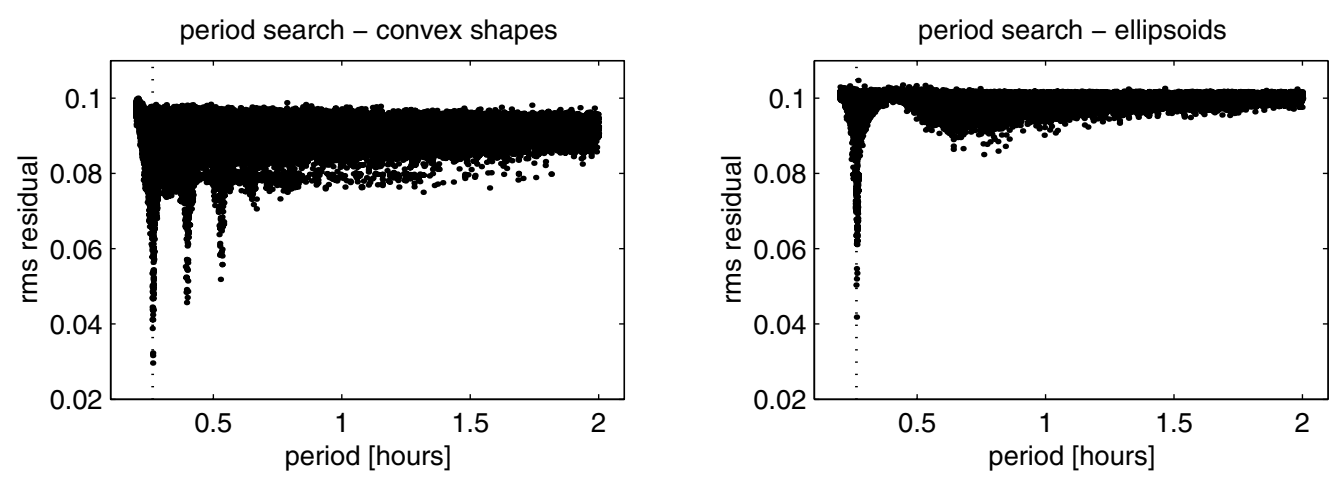

Figure 5. Period search for a fast rotator $(P=0.264 \mathrm{hr})$ using convex shapes (left) and triaxial geometrically scattering ellipsoids (right). The correct period is marked with the dotted vertical line.

model to compute synthetic brightness at given epochs covering ten years of observation. Gaussian noise of $3 \%$ was added to the data. The solution of the pole direction is very close to the correct value and the convex shape model is similar to the original.

\subsection{Fast rotators}

If we process sparse data of a fast rotating asteroid, then there is no solution found within the basic interval of typical rotation periods and it is necessary to look for the correct period outside this interval. The number of trial periods increases dramatically when scanning the short period range $(\sim 1000000$ trial periods for interval $0.2-2 \mathrm{hr}$ and 10 years of observation). Nevertheless, the simulations show that at least for PanSTARRS cadences even very short rotation periods can be detected.

A representative example of the period search for a fast rotator $(P=0.264 \mathrm{hr})$ is shown in Fig. 5. Both inversion algorithms - using convex shapes or ellipsoids - find the correct period. An important fact is that all trial periods from the basic interval $2-24 \mathrm{hr}$ give bad fit. There is only one period fitting the data well.

We assume that the rotation period is constant during the interval of observation. YORP effect that could be important at the time scale of several years for fast rotating small objects is in principle detectable from sparse data but no detailed simulations have been done so far.

\subsection{Slow rotators}

Another nonstandard case are slow rotators. Long periods can be scanned easily because the separation between subsequent trial periods increases with period. If we simulate photometric observations of an asteroid with the period $P$ which is longer than one day, there is usually a false period detection at $P / 2$ and some other harmonics (Fig. 6). However, the shape models corresponding to periods $P$ and $P / 2$ are usually completely different. The shape corresponding to the correct period almost always rotates around the principal axis of the moment of inertia (as does the original model). On the other hand, the shape corresponding to the half period is usually in a physically unacceptable rotation state - its rotation axis does not correspond with the principal axis of the moment of inertia. An example of the original shape, the model for the correct period, and the model for the half period is shown in Fig. 7. 

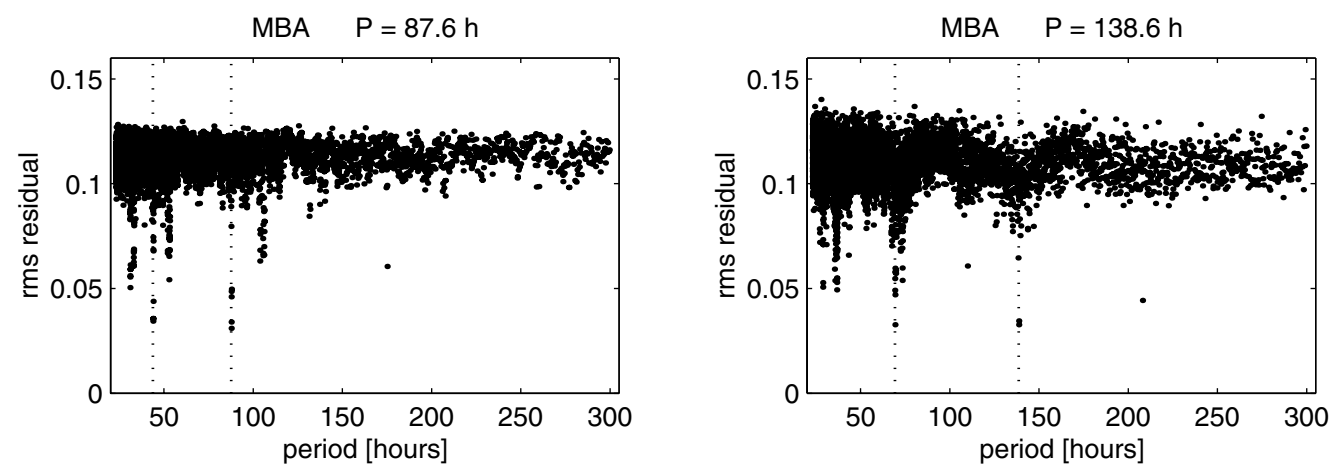

Figure 6. Period search for slowly rotating objects. Both the correct period and the half period fit the data well, they are marked with dotted lines.

\subsection{Binary asteroids}

Binary asteroids are an important part of the asteroid population. We carried out simulations with various binary systems in order to realize how the sparse photometry of such systems differ from that of single asteroids and what kind of results the formal application of lightcurve inversion to data of a binary system gives.

\subsubsection{Synchronous binary asteroids}

From a photometric point of view, a synchronous binary system behaves like a single body with one period of rotation. As has been shown by Durech \& Kaasalainen (2003) and Kaasalainen et al. (2002a), results of the lightcurve inversion procedure give us some indications about the binary nature of the target - a convex shape model of a binary asteroid exhibits large planar areas and the global shape is cone-like or brick-like. The same applies to the sparse data.

\subsubsection{Asynchronous binary NEAs}

We simulated sparse photometric data of asynchronous binary NEAs and tested the possibility of detecting such objects based on sparse photometric data. Our models of binary asteroids were based on physical properties of several objects derived by Pravec et al. (2006). A typical binary NEA consists of a fast rotating (2-3 hr), not very elongated primary (lightcurve amplitudes $0.08-0.2 \mathrm{mag}$ ), and of a secondary with the size $0.2-0.5$ of the primary. Secondaries are usually elongated (lightcurve amplitudes up to $0.8 \mathrm{mag}$ ) and their orbital periods are longer than $\sim 10 \mathrm{hr}$.

There are three effects important for the Pan-STARRS ability to detect binaries: the size of the secondary, the elongation of the primary, and the observing geometry. Mutual events are clearly visible for a big secondary and suitable geometry. If the secondary is small compared to the primary, its contribution to the brightness is small and the signal is dominated by the lightcurve of the primary.

The observing geometry is in many cases such, that there are no visible mutual events at all. The only clue that the object is 'nonstandard' is the fact that lightcurve inversion fails to find any fitting model for any single period.

Two examples of systems that could be recognized as binary from sparse data are shown in Fig. 8. Mutual events are visible in the brightness versus phase angle plot as drops in brightness and there is no single period model that would fit the data. Although sparse data are not sufficient for deriving a physical model, they can give us a strong 

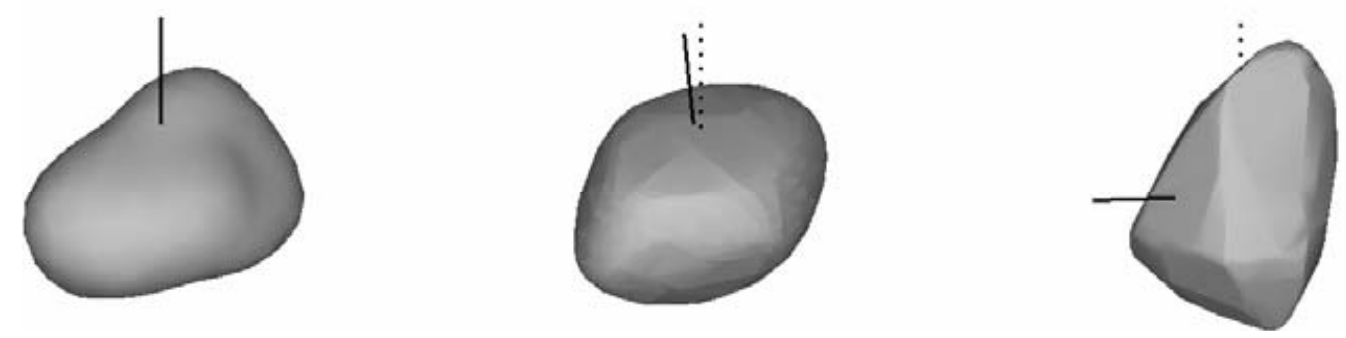

Figure 7. An example of shape reconstruction for a slow rotator. Rotation axis of the original shape (left), the shape model for the correct period (middle), and the model for the half period (right) is marked as a dotted line, while the principal axis of the moment of inertia is marked as a solid line. The axes coincide for the original shape.

indication that the object is binary. Such objects can be selected for further follow-up observations.

\subsection{Trans-Neptunian Objects (TNOs)}

TNOs create a special group of objects that differ from MBAs and NEAs. The observing geometry hardly changes due to their big distance from the Earth and from the Sun, and there are many spin/shape solutions corresponding to one period. Nevertheless, the correct period can be derived. There are often false solutions for other harmonic periods that fit the data only slightly worse than the correct period, but the shapes corresponding to those false periods are usually in a physically unacceptable rotation state (cf. Sect. 3.4 and Fig. 7) or have 'strange' triangular or hexagonal pole-on silhouettes.

\subsection{Tumbling asteroids}

We carried out several simulations with tumbling asteroids. Results of those simulations clearly show that sparse photometric data generated by a tumbler cannot be fitted with a principal axis rotator model, which means that tumblers cannot be misinterpreted as ordinary principal rotators. Although it is possible to carry out the inversion of lightcurves of a precessing asteroid and solve the inverse problem (Kaasalainen 2001), this cannot be done with sparse data. The parameter space of two periods is so huge that it is practically impossible to scan all combinations.

\section{Conclusion}

The era of sparse photometric data that is about to come will require automated processing of the data. With the large number of observed asteroids it will not be possible to deal with individual targets separately. The aim of our extensive simulations is to explore limits of sparse data sets and to understand results of lightcurve inversion applied to data produced by different types of asteroids. So far results show that sparse data can be sufficiently used for modelling of physical properties of asteroids. The main result is that sophisticated application of the lightcurve inversion method to sparse data does not produce false solutions. If a unique solution fitting data down to the noise level is found, it is always the correct solution. This enables us to process sparse photometric data automatically. All the cases that lead to multiple solutions that all fit the data well or such cases that cannot be fitted with any model can be removed from the processing pipeline and tagged for closer investigation and photometric follow-up. 

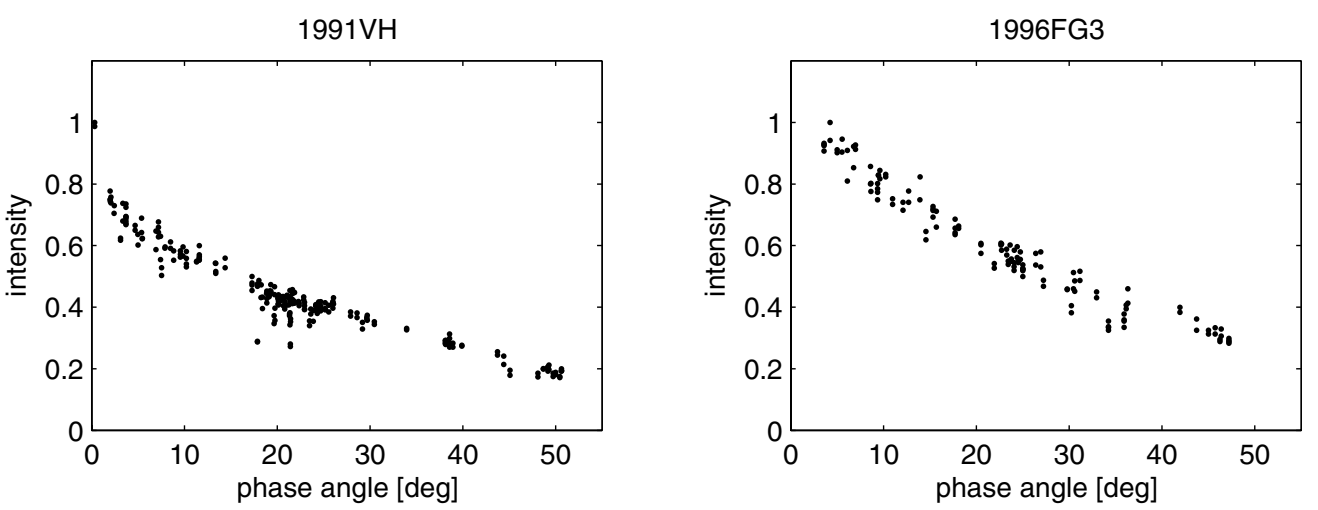

Figure 8. Intensity against the solar phase angle plot for two asynchronous binary NEAs. The decrease of intensity due to the mutual events is visible in both cases. The size ratio of the secondary to the primary is $\sim 0.4$ and $\sim 0.3$ for $1991 \mathrm{VH}$ and 1996FG3 respectively. Both primaries are nearly spheroidal objects.

Photometric follow-up observations of selected targets (suspected binaries or tumblers, extreme fast or slow rotators, etc.) will be very important. Only a few dense lightcurves can reveal the nature of such object. As is shown in KD, Sect. 4.1, inversion of combined datasets can lead to a unique physical model even if the inversion would not be possible with either dataset alone. Sparse sequences that contain few points or are too noisy to be used alone can be combined with ordinary lightcurves and a model can be derived. We can expect hundreds or thousands of asteroid models within next few years if we combine the first Pan-STARRS data with the already available database of dense lightcurves for about 2000 asteroids. Tens of thousands of models will be available within the next decade after Pan-STARRS and other surveys collect enough data.

\section{Acknowledgements}

This work was supported, in part, by CIMO and the Academy of Finland. We thank for the computer time on the computational cluster Tiger at the Astronomical Institute of the Charles University in Prague, and we thank L. Šubr, who has designed and maintained the cluster.

\section{References}

Durech, J. \& Kaasalainen, M. 2003, Astron. Astrophys. 404, 709

Ďurech, J., Grav, T., Jedicke, R., Kaasalainen, M. \& Denneau, L. 2006, Earth, Moon \& Planets, in press

Kaasalainen, M. 2001, Astron. Astrophys. 376, 302

Kaasalainen, M. 2004, Astron. Astrophys. 422, L39

Kaasalainen, M. \& Lamberg, L. 2006, Inverse Problems 22, 749

Kaasalainen, M., Pravec, P., Krugly, Y. N., Šarounová, L., Torppa, J., Virtanen, J., Kaasalainen, S., Erikson, A., Natheus, A., Durech, J., Wolf, M., Lagerros, J. S. V., Lindgren, M., Lagerkvist, C.-I., Koff, R., Davies, J., Mann, R., Kušnirák, P., Gaftonyuk, N. M., Shevchenko, V. G., Chirony, V. G. \& Belskaya, I. N. 2004, Icarus 167, 178

Kaasalainen, M. \& Torppa, J. 2001, Icarus 153, 24

Kaasalainen, M., Torppa, J. \& Muinonen, K. 2001, Icarus 153, 37

Kaasalainen, M., Torppa, J. \& Piironen, J. 2002a, Astron. Astrophys. 383, L19

Kaasalainen, M., Torppa, J. \& Piironen, J. 2002b, Icarus 159, 369

Kaasalainen, S., Kaasalainen, M. \& Piironen, J. 2005, Astron. Astrophys. 440, 1177 
Marchis, F., Kaasalainen, M., Hom, E., Berthier, J., Enriquez, J., Hestroffer, D., Le Mignant, D. \& de Pater, I. 2006, Icarus 185, 39

Muinonen, K. 1996, Earth, Moon \& Planets 72, 339

Pravec, P., Scheirich, P., Kušnirák, P., Šarounová, L., Mottola, S., Hahn, G., Brown, P., Esquerdo, G., Kaiser, N., Krzeminski, Z., Pray, D. P., Warner, B. D., Harris, A. W., Nolan, M. C., Howell, E. S., Benner, L. A. M., Margot, J.-L., Galád, A., Holliday, W., Hicks, M. D., Krugly, Y. N., Tholen, D., Whiteley, R., Marchis, F., Degraff, D. R., Grauer, A., Larson, S., Velichko, F. P., Cooney, W. R., Stephens, R., Zhu, J., Kirsch, K., Dyvig, R., Snyder, L., Reddy, V., Moore, S., Gajdoš, Š., Világi, J., Masi, G., Higgins, D., Funkhouser, G., Knight, B., Slivan, S., Behrend, R., Grenon, M., Burki, G., Roy, R., Demeautis, C., Matter, D., Waelchli, N., Revaz, Y., Klotz, A., Rieugné, M., Thierry, P., Cotrez, V., Brunetto, L. \& Kober, G. 2006, Icarus 181, 63

Torppa, J., Kaasalainen, M., Michalowski, T., Kwiatkowski, T., Kryszczyńska, A., Denchev, P. \& Kowalski, R. 2003, Icarus 164, 346 